\title{
El escenario político del campo de las artes de la Universidad de Sonora
}

\author{
Dr. Leonel de Gunther Delgado \\ Universidad de Sonora \\ Departamento de Bellas Artes \\ Igunther@capomo.uson.mx
}

\begin{abstract}
Hay política porque el hombre es el ser vivo que, en el lenguaje, separa la propia nuda vida y la opone a sí mismo, y, al mismo tiempo, se mantiene en relación con ella en una exclusión inclusiva.
\end{abstract}

Giorgio Agamben

Durante milenios el hombre siguió siendo lo que era para Aristóteles: un animal viviente y además capaz de una existencia política; el hombre moderno es un animal en cuya política está puesta en entredicho su vida de ser viviente

Michel Foucault

\section{Introducción}

En otro trabajo, abordamos la delimitación del campo de conocimiento de las artes de la Universidad de Sonora argumentando la necesidad de un esfuerzo de clarificación de las orientaciones teóricas y metódicas para su demarcación (De Gunther, 2015).

Tal clarificación involucró el tejido de diferentes propuestas que aluden a la noción de campo de conocimiento y, también, de aquellas que, por su cercanía y relevancia, deberían ser consideradas. Nos referimos a las nociones de "Campo de conocimiento" (Bunge, 1999), de "Campo" (Bourdieu, 1993, 1999) y de "Campo de excepción" (Agamben, 1998, 2006, 2007). Cada una de ellas fue traducida como una dimensión de estudio para explicar y comprender el campo de las artes de la Universidad de Sonora.

Así, la noción de Campo de conocimiento la traducimos como la dimensión formal de estudio, a través de ella articulamos las diferentes variables que permiten evaluar su consolidación o su exigua formación. La segunda, Campo, la traducimos como la dimensión social, útil para el reconocimiento de los agentes que intervienen en la configuración y constitución del campo. La tercera, Campo de excepción, la traducimos como la dimensión política del estudio, a través de ella, damos cuenta de la creación de normas de excepción que alejan al campo de los ideales de la investigación científica.

Reconocimos, además, que tal planteamiento sólo adquiere sentido pleno en la medida en que el campo de conocimiento de las artes sea contextualizado en un mundo en proceso de transformación, en donde la aparición y desaparición de fenómenos ocurre de manera permanente y el replanteamiento de las nociones de trabajo y subjetividad, obligan al replanteamiento de nuestras formas de conocer. Desde la lógica anterior, el propósito principal de este trabajo consiste en pre- 
sentar el bosquejo del escenario político del campo de conocimiento de las artes de la Universidad de Sonora. En particular, a través de la operacionalización de la noción de autoridad. Si bien, nuestra contribución se centra en el ámbito teórico, se aportan datos empíricos que permiten la clarificación y desarrollo conceptual de la dimensión política del campo, es decir, de su traducción a su aplicación en la práctica discursiva de sus miembros. Para ello, seguimos una metodología cualitativa, en la cual, a través de la técnica de entrevista a profundidad, se recogen informaciones de miembros representativos del campo de las artes de la Universidad de Sonora.

Los hallazgos parciales que ahora presentamos, permiten afianzar la hipótesis de trabajo, en la cual sugerimos que las profundas transformaciones que experimentamos hoy en la escala global, en particular la nueva subjetividad y el trabajo exige un replanteamiento de las formas epistémicas para el conocimiento del campo de conocimiento de las artes de la Universidad de Sonora, una visión tejida en conjunto para su estudio.

\section{La dimensión política del cam- po de las artes}

La noción de campo de conocimiento, campo, y campo de excepción encuentra diferentes acepciones sea en la filosofía (Bunge, 1999), en la sociología (Bourdieu, 1993, 1999) o en la política (Agamben, 1998, 2006, 2007). La primera de ellas, Campo de conocimiento, articula diferentes variables que permiten evaluar su consolidación o su exigua formación. La segunda, Campo permite reconocer de los agentes que intervienen en la configuración del campo. La tercera, Campo de excepción, da cuenta de la creación de normas de excepción que alejan al campo de los ideales de la investigación científica. Se trata, como hemos señalado en otro lugar (De Gunther, 2015), de variables para evaluar la configuración del campo de conocimiento; para reconocer a los agentes que lo configuran y lo constituyen y para el reconocimiento de rasgos de excepción que, como dispositivos, encarnan y cercan el campo para su producción, organización y distribución de conocimiento. Es aquí donde se pone en juego la distinción entre vida, por el solo hecho de estar vivo, zoé y vida como dimensión de la existencia política, bios. Una distinción que ha permanecida oculta y funciona como el paradigma del siglo XX y se extiende hacia el XXI.

La traducción de estas nociones de campo de conocimiento, campo y campo de excepción como dimensiones formal, social y política y articuladas en red, aportan novedad para comprender de la manera menos determinista, menos reductora y más articulada posible el campo de conocimiento de las artes de la Universidad de Sonora. Tal tejido permite reconocer el campo de conocimiento de las artes como el despliegue, la estabilización y des-estabilización (Latour, 2008) del mismo, en una especie de re-configuración permanente como campo. Se trata también del orden-desorden-organización, enunciado en las ciencias físicas (Prigogine, 2000) $\mathrm{y}$ traducido a las humanidades y las ciencias sociales como Orden-desorden-organización (Morin, 2008).

Esta traducción, colocada en un contexto e-norme, donde la presencia de un conjunto de elementos intensificadores desfigura y transforma la sociedad existente sea a través de la erosión y horadamiento del mundo moderno, del desorden, el azar y la incertidumbre, por un lado, y por el otro, la presencia simultánea del orden, el determinismo y la certidumbre sea la primera como ciencia emergente y la segunda normal, configuran una visión doble de un sistema-mundo complejo, después del liberalismo (Bryant, 2007; De Landa, 2011; Kuhn, 1977; Lanceros, 2006; Wallerstein, 1996, 2007). Factores que, como lo ha señalado Motta (2013), 


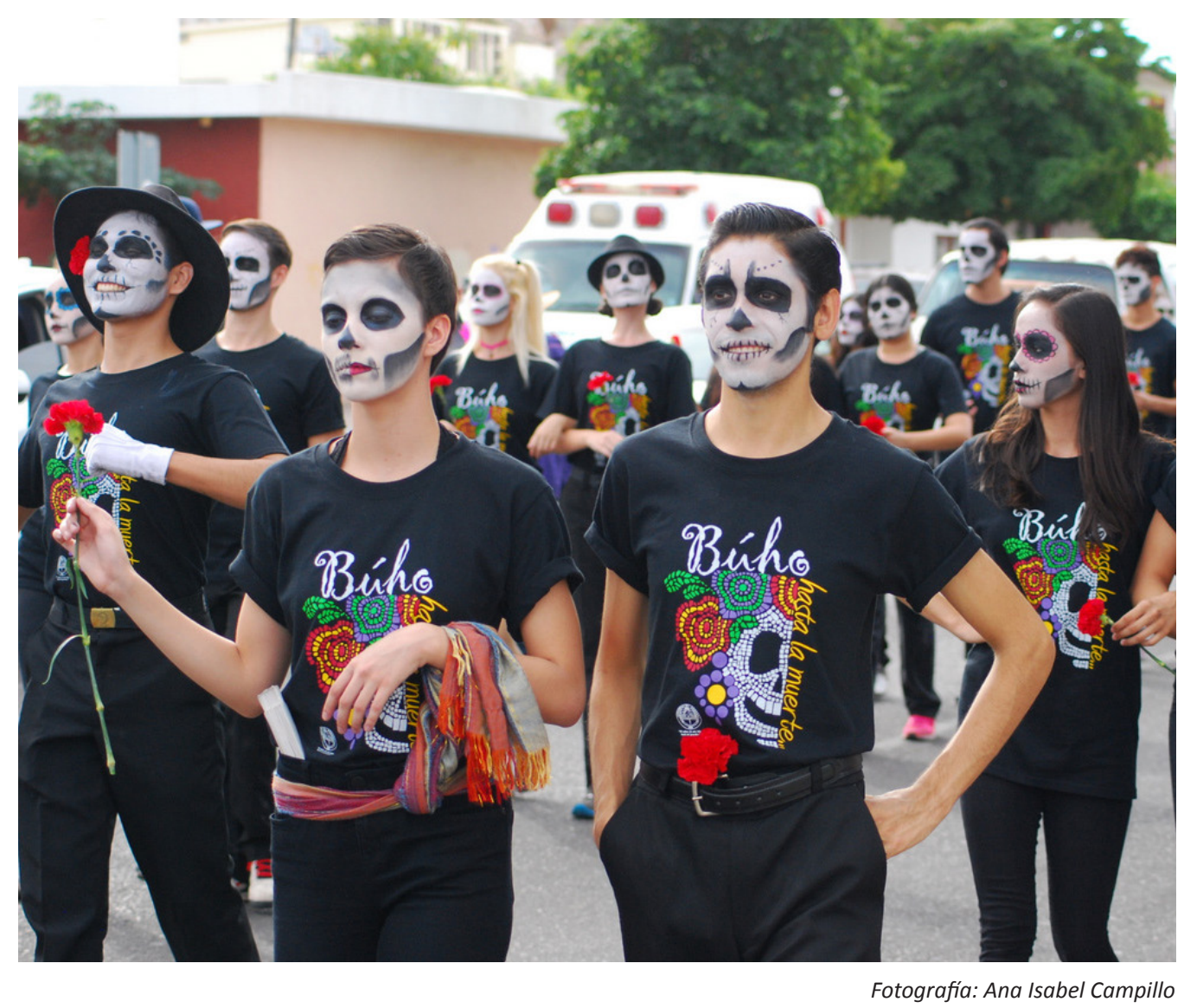

contribuyen en la transformación social. Se trata de procesos de diferenciación que conducen a la fragmentación social; de procesos de pérdida de tradiciones que genera y regenera crisis de identidad y desarraigos; de la articulación social débil que genera anomia; de la exigencia de múltiples roles de los actores sociales, generando paradojas éticas y del crecimiento exponencial de los conocimientos, en cuyo caso, pone en crisis la organización del conocimiento (Comunicación personal, mayo 2013).

Si bien, tales procesos buscan mantener la viabilidad organizativa del sistema por medio de clausuras o aperturas, sea a través de su estructura o función, también es cierto que, en su operar, generan tensiones polares, que hacen emerger nuevas configuraciones imaginarias de lo social. En este sentido, tales transformaciones también inciden en la constitución del campo de conocimiento. Las nuevas for- mas en que se nos presenta hoy el trabajo, traducidas en red, consorcios, "bunkers" o "brazileñización", aunado a las transformaciones en la subjetividad que inciden en la fragmentación social, el debilitamiento de las tradiciones o desarraigo, también pone en crisis la organización del conocimiento a través de los propios miembros inscritos en los campos de conocimiento.

Desde esta lógica planteamos aquí la dimensión política del campo de las artes de la Universidad de Sonora, para ello, seguimos las ideas de Giorgio Agamben, un pensador colocado en la línea post-marxista, que cuestiona la naturaleza reduccionista y antidemocrática del pensamiento marxiano, así como cualquier explicación del cambio en términos de clases sociales específicas. Tal corriente acepta las ideas de la acción política marxiana; pero niega el énfasis determinante de la economía o de clases universales. El 
post-marxismo ofrecen argumentos para una democracia radical (Lechte, 2008).

\section{El campo de conocimiento de las artes como proceso político de inclusión-exclusión}

Agamben (1998, 2006, 2007), deriva la noción de campo de excepción de la confluencia entre el modelo jurídico-institucional y el modelo biopolítico del poder, el cual constituye el núcleo oculto del poder soberano. Es una noción inscrita dentro de la lógica jurídica, política y filosófica del mismo. Presenta conceptos clave como zoé, vida desnuda y bios, vida pública o política, así como bio-política para el desarrollo de su discusión. Tal planteamiento nos interesa aquí para dimensionar la política del campo de las artes.

El campo, señala Agamben, es el espacio que se abre cuando el estado de excepción comienza a devenir en regla. En ese momento, el estado de excepción, que era esencialmente una suspensión temporal del ordenamiento, adquiere un orden especial permanente que, como tal, permanece, sin embargo, constantemente fuera del ordenamiento normal. Una relación paradójica que, dentro del campo de las artes, instituye una norma de operación o ley, en un momento en el que se coloca fuera o como excepción su propio operar como política de conocimiento: "El campo es la estructura en la cual el estado de excepción, sobre cuya posible decisión se funda el poder, viene realizado en forma estable" (Agamben, 1998).

Agamben (2006), al problematizar acerca de la conceptualización de Schmidt sobre la soberanía, pone de manifiesto la investidura del soberano, la cual se hace presente al decidir sobre el estado de excepción. El soberano es quien decide el estado de excepción. Tal atributo crea un doble vínculo: le permite simultáneamente estar fuera y dentro del sistema jurídico. Es a la vez vida y norma, hecho y derecho.
El estado de excepción es el momento donde emerge claramente la ambigüedad de la simultaneidad entre inclusión-exclusión, y es a la vez "el dispositivo que debería mantener unidos a los elementos contradictorios del sistema jurídico" (pp. 14-15), un límite entre la doctrina del Estado y del derecho, "limita con la esfera de la vida y se confunde con ella" (p. 22). De ahí que, el autor que comentamos, retome la octava tesis benjaminiana, según la cual el estado de excepción es la regla en que vivimos. El estado de excepción suspende el derecho para garantizar su continuidad y su existencia. Es una suspensión temporal que ha devenido en norma durante el siglo XX.

Una de las paradojas del estado de excepción quiere que sea imposible distinguir en él entre la transgresión de la ley y su ejecución, de manera que lo que es conforme a la norma y lo que la viola, coinciden sin fisuras (quien pasea durante el toque de queda no está trasgrediendo la ley en mayor medida de lo que la está cumpliendo el soldado que, eventualmente, le mata) (Agamben, 2006, p. 78).

El campo de concentración, como puro, absoluto e insuperado coincide con el "espacio biopolítico (fundado en cuanto tal exclusivamente en el estado de excepción), aparece como el paradigma oculto del espacio político de la modernidad, del que tendremos que aprender a reconocer las metamorfosis y los disfraces" (Agamben, 2006, p. 156).

De ahí que lo característico de la política moderna sea para Agamben (2006),

... el hecho de que, en paralelo al proceso en virtud del cual la excepción se convierte en regla, el espacio de la nuda vida [vida desnuda] que estaba situada originariamente al margen del orden jurídico, va coincidiendo de manera progresiva con el espacio político, de forma que exclusión e inclusión, externo e interno, bios y zoé, derecho y hecho entran en una zona de irreductible indiferenciación (pp. 18-19). 
La anulación del proceso de diferenciación convierte a la vida desnuda, zoé como "el sujeto y el objeto del ordenamiento político y de sus conflictos, el lugar único tanto de la organización del poder estatal como de la emancipación de él" (p. 19).

Esta paradoja se asienta en las distinciones entre vida como zoé y vida como bios. En el primero, Agamben reconoce la vida desnuda en los asilados, en los buscadores de refugio y en la muerte cerebral, todos tienen en común la vida humana, pero carecen de la protección de la ley y, la muerte cerebral en particular, permanece en calidad de paisaje vivo, a través de la interpretación científica (como se cita en Lemke, 2011, p. 55); mientras que bios, según el planeamiento de Agamben (2006), alude a la vida en común, a la existencia política sea como libertad y finalidad y, además, a la creación de una forma de vida en la ciudad, un forma de vida de los individuos en la plaza pública. Los conceptos clave de esta discusión juegan un papel decisivo en la configuración del campo de excepción. La excepción es la que funda el campo de la biopolítica moderna, el control de la vida sin límite. Así los términos griegos de vida desnuda y de existencia política y finalidad constituyen el andamiaje de la política y también de la política de los campos de conocimiento. Vale decir, que no es lo mismo el conocimiento de la política que las políticas del campo de conocimiento. El término biopolítica, fundado sobre la excepción, no es su única acepción, según Lemke (2011), evoca múltiples sentidos, es un término usado hoy no sólo por expertos sino empleado en diferentes disciplinas y discursos sea para discutir políticas de asilo político, prevención del SIDA, regulaciones sobre el aborto. Asimismo, está ligado a la toma de decisiones racionales y a las organizaciones de la vida social, así como también, en sentido opuesto, a la eugenesia y al racismo. En términos ideológicos se ha ligado tanto a la vieja derecha y ultraderecha como la nueva izquierda y es empleado tanto por defensores del progreso biotecnológico como por marxistas comprometidos y racistas intolerantes. También alude a la idea de "un más allá de la vida biológica, se trata de las acciones y de la toma de decisiones comunes, de la necesidad de incorporar la experiencia y los hechos biológicos y abrirlas a al reino de la libertad y de la interacción humana. Los principales expositores del tema, siguiendo y separándose de la herencia de Foucault son, además de Agamben, Negri, Heller, Giddens, Rabinows, entre otros.

\section{Quién es la autoridad en el campo de las artes}

El recorte analítico que encontramos para el estudio de la dimensión política del campo de conocimiento de las artes, lo derivamos de la discusión sobre la dificultad del concepto de auctoritas [autoridad] desarrollado por Agamben (2007). Señala que se refiere a una fenomenología jurídica amplia ligada tanto al derecho público como al privado: "la autoridad es una propiedad de la persona-auctor-que interviene para conferir validez jurídica al acto de un sujeto que por sí solo no puede llevar a cabo un acto jurídico válido" (p. 140).

La discusión se centra aquí en la propiedad de la persona que interviene para otorgar, aumentar o conferir validez al acto. Desde este punto de vista, continua Agamben, la autoridad necesita una relación entre dos elementos, la autoridad que manifiesta su propiedad a través del autor que valida y aquel que toma la iniciativa del acto.

Tal realización no proviene, y esto es paradójico, de la facultad o propiedad conferida a la autoridad, sino que el poder del autor proviene de su condición, en este caso de pater o mater, según sea el caso. Así, siguiendo a Agamben la norma "puede aplicarse al caso normal y puede 
ser suspendida sin anular integralmente el orden jurídico, porque en la forma de autoridad o de la decisión soberana ella se refiere inmediatamente a la vida, surge de ella" (2007, p. 154). De ahí que distinguir vida y derecho, anomalía y ley coincida con la máquina biopolítica, con los mecanismos de control emergente sea el reconocimiento de retina o los teléfonos celulares los cuales no crean subjetividad, sólo control.

Indagar en el campo de conocimiento de artes quién es la autoridad, permite precisar algunas ideas clave que configuran esa dimensión. La noción de autoridad como "soberano", parece ligarse en el modo de operación de los campos. No sólo se controla la producción, organización y distribución del conocimiento, sino también la vida al interior de ellos.

En este sentido, el campo de las artes como política del conocimiento parece comportarse como si marcara una excepción de normas e ideal de conocimiento, donde la distinción entre el adentro y el afuera, que demarca la parcela de estudio, pareciera constituir una zona de escasa diferenciación, en la cual los miembros y los posibles miembros no contienen mediación alguna al momento de su incorporación o garantías de permanencia. Es decir, nos encontramos ante una estructura, cuya excepcionalidad lo coloca al margen de las reglas del conocimiento, no evaluamos aquí su pertinencia o impertinencia, lo que queremos resaltar es la descolocación del campo de las artes en la dinámica productiva, organizativa y de producción del conocimiento, en sus formas de operación.

El control de la vida desnuda encuentra aquí un terreno de indiferenciación. No se trata de un momento para la existencia pública colectiva en la plaza pública del conocimiento, sino del control del cuerpo y con ello de la cognición. La autoridad es quien decide y en su decisión crea un doble vínculo que le permite estar dentro y fuera del sistema. Es, simultáneamente, vida y norma, hecho y derecho.

Tenemos una autoridad en una disciplina artística, se ha instalado como modelo y también como autoridad política para las autoridades políticas (a lo mejor ni lo saben las autoridades). Esa autoridad establece el conjunto de prácticas, entras al campo y te doy estabilidad ... esa autoridad, al haberse instalado con esa legitimidad... aborda la vida y eso hace que se haga lo que se dice. En el sustrato del pensamiento del profesor que ha entrado al campo, debe haber un pensamiento estético que ya no responde a la necesidad de la época y lo acepta.... (E001) ${ }^{1}$

La dimensión política cerca la vida profesional a través de crear normas no establecidas, que construyen formas de expulsión y a la vez de inclusión del campo. Una construcción de paisajes vivos, regulados por la interpretación:

Hay un abuso de poder y control de la autoridad... el caso es que hacen que profesoras y alumnas de la escuela salgan del mismo, por poner en entredicho a la autoridad. Hablan contigo como profesor y te dicen las reglas que hay que seguir para tratar con ellas... mira nomas, le dije a la maestra que me dijo, desde entonces ya no doy clases ahí y ellas no pueden trabajar en lo que estudiaron (E003).

La autoridad se encuentra distribuida en "el artista... en la institución o instituciones" (E002), Incluso “están también en las instancias nacionales y estatales: CENIDIM de Bellas Artes, el Instituto Sonorense de Cultura ..." (E003), ahí se decide la validez del acto, sea como inclusión de contenido o de vaciamiento del mismo, por ejemplo, "el FAOT (Festival Internacional Alfonso Ortiz Tirado,

\footnotetext{
${ }^{1}$ Con E001, E002, E003... aludimos a la identificación de los entrevistados
} 
realizado en el estado de Sonora, México), surge como promotor de la ópera, en las últimas fechas se han introducido cuestiones populares o populistas donde se presenta una fiesta para una ciudad: alta y baja cultura... no sé cómo decirlo bien. O, por ejemplo, Las fiestas del Pitic (Festival realizado en la capital de Sonora, México), tienen un sentido lúdico, el consumo es diverso, lo comercial, lo clásico, lo tradicional y son en todas las artes...Una fiesta global (E003).

"A mí me sorprende el apoyo que se les da al trabajo de ciertos grupos que musicalizan obras de la literatura desde la tradición norteña, la institución piensa que eso es rescate musical y lo avala, lo demás no existen ni tiene futuro" (E003). "La política sigue siendo, lamentablemente, un elemento crucial donde las prebendas o amistades generan el posible apoyo.... Podemos ver auges de áreas específicas" (E002).

También se trata de un esfuerzo de construir diferenciación, aunque esta sólo se logre en la parte de la racionalidad instrumental, la trasgresión de la norma se da tanto en el que la aplica como en el que la vigila:

Hay un comité por ahí... Que decide ahí... Esa es la autoridad. Pero nosotros estamos en un tren de análisis donde nos preguntamos cómo son reconocidos nuestros logros, nuestros proyectos. Nuestras investigaciones deben tener otro reconocimiento... no entramos en el Sistema Nacional de Investigadores, no podemos entrar al Sistema Nacional de Creadores, hay que ser creador, compositor, para entrar ahí, hay muchas trabas... al ejecutante no se le considera... pero los compositores la tienen en chino ... Uno va generando esas redes de colaboración para producir... Se conjuntan las condiciones. A pesar del valor educativo que ves, la cultura se institucionaliza y se echa a perder... las personas terminan siendo las instituciones... aquí lo que yo digo es la ley.... Esa cerrazón se ha ido dando todo el tiempo... (E002).
El campo de las artes es un acontecimiento político, que marca el espacio de la política del conocimiento del campo de las artes, un regulador de la vida intelectual y simbólica para la producción de conocimiento, es pues una máquina -aunque la metáfora sea un tanto parcial- para la producción de contenidos: un texto, como señala Bhaskar (2014), como una obra musical e incluso una puesta en escena, son contenidos y todos son publicables, para ello, habría que reconsiderar nuestra posición con respecto al soporte y al contenido, para evitar una posible confusión.

Estamos frente a un campo de conocimiento que requiere de un complejo entramado para su explicación y comprensión. Hay política, como se lee en el epígrafe escrito al margen del texto, "porque el hombre es el ser vivo que, en el lenguaje, separa la propia nuda vida y la opone a sí mismo, y, al mismo tiempo, se mantiene en relación con ella en una exclusión inclusiva" (Agamben, 2007, p. 20).

En cuanto a las valoraciones estéticas de los cuerpos, por ejemplo, si nombro el vendado [se refiere a la persona que baila la danza del vendado en el noroeste de México], ser un venado es ser esbelto, atlético... esa idea la instala Amalia Hernández con un cuerpo de bailarín clásico. Sin embargo, yo nombro y digo que el venado puede ser gordo o flaco, así como en las comunidades de acá (se refiere al estado de Sonora en México), lo importante es la interpretación, pero la autoridad política de la disciplina dice, que esos no son vendados, el venado es el de Amalia Hernández... (E001).

Tal control también toca al cuerpo "Porqué caminas tan feo en el escenario... se toma conciencia de que soy un personaje... párate derecho, no mires al piso, vete con determinación a tocar..." (E002). "Se vieron muy bien en lo contemporáneo, y se movieron según sus posibilidades, le dije a R. Es lo que les digo 
a mis compañeras, responde R., ¿Por qué si ellas tienen un cuerpo así porque les piden un cuerpo que no es suyo, si ellas están bailando?” (E001).

La descolocación, un fenómeno que se recorre como territorialización y desterritorialización, constituye un fenómeno que no puede quedar al margen de la comprensión del campo de las artes, si bien puede confundirse con la dimensión social, la distinción no la hacemos en el sentido de los condicionamientos objetivos de la existencia del campo, si lo hiciéramos sería imposible hablar acerca de la dimensión política del campo, lo que resaltamos aquí es la oposición entre la singularidad y la autoridad en las relaciones del campo, el individualismo es la excepcionalidad de la regla, que se ha convertido en la regla y restituye autoridad al artista.

Las instituciones toleran el campo, lo permiten, porque los que lo dirigen son puestos políticos, encontrar a un funcionario con capacidad, sensibilidad, con cultura, es raro, sería una rareza encontrar a un Vasconcelos.... encontrar a un político que entienda el arte seria rarísimo. La autoridad representa las personalidades que están en las instancias públicas... definen el criterio de autoridad. Pero la autoridad tampoco está en la escuela, la escuela no educa la sensibilidad. Se queda a nivel de telenovela a nivel de cursilería... no me gusta Bach, me gusta el Gallo Elizalde (Se refiere a un cantante de banda mexicano), no tiene mal gusto... tiene mala educación...Su música es para gente culta, no es para gente sensible. Así es el artista quien debería ser la autoridad. Cuando la obra se convierte en obra... cuando se comunica algo es cuando se da la comunicación entre el artista y el que lo ve.... El pensamiento cotidiano del artista no resuelve problemas. Han glorificado artistas con enfermedad... cuando tengo esta enfermedad no puedo ser artista, que voy a hacer, qué voy a decir, tiene que salir de su cotidianidad... (E004).
Tal afirmación ofrece un paralelo entre las nociones de clausura o apertura que, como estrategias de conservación o transformación de un campo, constituyen prácticas específicas al interior del mismo, guarda un mismo paralelo con la noción de institucionalización que cerca las posibilidades de incorporar lo emergente. Surge aquí la noción de norma y anomalía, como parte de la distinción de quien puede declarar el estado de excepción en el campo y operar en esa ambigüedad.

\section{Conclusiones}

Establecer las distintitas dimensiones para estudiar el campo de las artes de la Universidad de Sonora constituye un esfuerzo para re-ligar el conocimiento disperso y disponible en las disciplinas, para articularlo en forma de redes y mantener abierta la comprensión de lo social a través de la práctica y la reinvención permanente del mito de Sísifo en el ámbito académico de las humanidades y las artes. Es a la vez, una posición intermedia, "Hay que ser sobrio con el poder" escribe Latour (2008), para referiste a la idea de que no debe haber explicaciones poderosas sin controles y equilibrios (p. 363).

La presentación parcial de la dimensión política del campo de las artes pone en juego la construcción de reglas excepcionales que configuran su operar, una ficción que se pone de manifiesto en la paradójica realización del campo de excepción, que imposibilita la distinción entre la transgresión de la ley y su ejecución, de manera que lo que es conforme a la norma y lo que la viola, coinciden sin fisuras (Agamben, 2006). También ofrece pautas para dar cuenta de los factores que dinamizan e intensifican los procesos de transformación, en cuanto a la anomalía o anomías que generan la disolución de los vínculos sociales y la generación de desarraigo en los miembros.

Tres aspectos nos parecen claros, aunque debemos continuar con esta exploración. (1) La figura de la autoridad, como ana- 
lítica del campo, constituye un elemento útil para comprender esta dimensión. Se refiere a un hecho positivo, que da pauta para apuntalar la idea de que la singularidad de la autoridad, como excepción, es ahora la norma que opera en un estar dentro y fuera del campo.

(2) Resulta difícil distinguir entre la norma y la anomalía, en el hecho y el derecho, como propiedad del pater o mater porque la forma de la decisión soberana se refiere inmediatamente a la vida, surge de ella. El discurso del profesorado está volcado en la doble dimensión de la vida, sea como vida desnuda o como existencia política, con escasa diferenciación entre ambas, esa característica de exclusión inclusiva.

(3) La creación de paisajes vivos como resultado del operar del campo en su excepcionalidad, la reducción de los sujetos a la vida desnuda, es una de las consecuencias más graves del mismo, que sugieren la imperiosa necesidad de seguir trabajando en su elucidación. El hombre moderno, como lo ha dicho Foucault, es un animal en cuya política está puesta en entredicho su vida de ser viviente

\section{Bibliografía}

Agamben, G. (1998). ¿Qué es un campo? Retrieved from http://www.egs.edu/faculty/giorgio-agamben/articles/que-es-campo/ website:

Agamben, G. (2006). Homo sacer. El poder soberano y la nuda vida I (A. Cuspinera, Trans.). Valencia: Pre-textos.

Agamben, G. (2007). Estado de excepción. Homo sacer, II, I (F. Costa \& I. Costa, Trans. 1a ed.). Buenos Aires: Adriana Hidalgo editora.

Bhaskar, M. (2014). La máquina de contenido. Hacia una teoría de la edición desde la imprenta hasta la red digital (R. Rubio, Trans.). México: Fondo de Cultura Económica.

Bourdieu, P. (1993). La lógica de los campos. Zona Erógena, 16.

Bourdieu, P. (1999). Meditaciones pascalianas (T. Kauf, Trans. 1a ed.). Barcelona: Editorial Anagrama.

Bryant, A. (2007). Modernidad líquida, compeljidad y turbulencia (F. Ochoa de Michelena, Trans.). In Z. Bauman \& F. Ochoa de Michelena (Eds.), Arte, ¿̇líquido? (1era ed., pp. 5970). Madrid: Sequitur.

Bunge, M. (1999). Buscar la filosofía en las ciencias sociales (T. Aguilar., Trans.). México: Fondo de Cultura Económica.

De Gunther, L. (2015). Aportes para el abordaje del campo de conocimiento de las artes de la Universidad de Sonora: las dimensiones formal, social y política de su constitución. In C. Hurtado, R. Lozano, L. De Gunther \& U. Mazariegos (Eds.), Una Visión Interdisciplinaria del Arte (1a ed., pp. 44-62). México: Universidad de Sonora.

De Landa, M. (2011). Philosophy and simulation: the emergence of synthetic reason. New York: Continuum International Publishing Group.

Kuhn, T. (1977). Essential Tension. Selected Studies in Scientific Tradition and Change (1a ed.). Chicago: The University of Chicago Press.

Lanceros, P. (2006). All that is solid. Política(s) de la globalización. In A. Ortíz-Osés \& P. Lanceros (Eds.), La interpretación del mundo. Cuestiones para el tercer milenio (1a ed., Vol. 23, pp. 11-42). Barcelona: Antrophos-UAM.

Latour, B. (2008). Reensamblar lo social. Una introduccion a la teoría del actor-red (G. Zadunaisky, Trans.). Buenos Aires: Manantial.

Lechte, J. (2008). Fifty Key Contemporary Thinkers. From Structuralism To Post-Humanism (2a ed.). New York: Routledge. Taylor \& Francis Group. 
Lemke, T. (2011). Biopolitics an advanced introduction (E. Trump, Trans. 1a ed.). New York: New York University Press.

Morin, E. (2008). Complejidad restringida y complejidad generalizada. Universidad Autónoma de Nuevo León: Catedra Itinerante Edgar Morin (Unesco)-IIPC-USAL-UANL.

Prigogine, I. (2000). El tiempo y el devenir a partir de la obra de llya Prigogine. Actas del coloquio internacional de 1993. (J. Álvarez, Trans. 1era 1996 ed.). Barcelona: Gedisa- Coloquio de Cerisy.

Wallerstein, I. (1996). Después del liberalismo (S. Mastrángelo, Trans. 1a ed.). México: Siglo XXI editores-CIICYH-Unam.

Wallerstein, I. (2007). Impensar las ciencias sociales. Límites de los paradigmas decimonónicos (S. Guardado, Trans. 5a ed.). México: Siglo XXI editores.

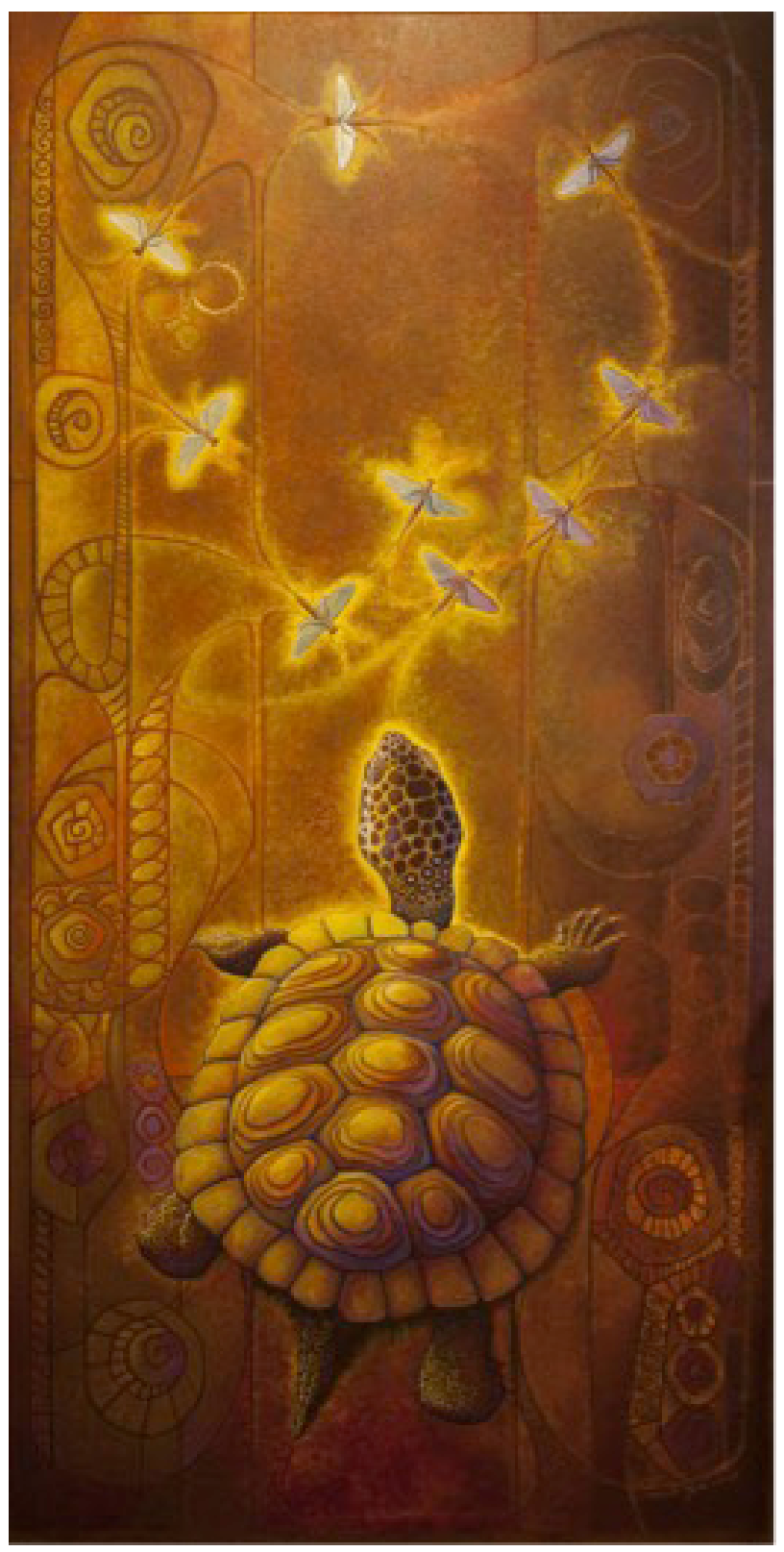

